\title{
The open learning initiative: \\ New directions for higher education
}

\author{
Bruce King \\ University of South Australia
}

I must begin with a confession. This paper is not about educational technology. Its concern is the Open Learning Initiative (OLI), which may well prove to be the most significant intervention in post secondary education of the Hawke and Keating Labor Governments, eclipsing even the creation of the post-1987 'new' universities and the Unified National System of higher education. My presentation today is not entirely unrelated to your concerns, however. In my view, the OLI has at least some of its origins in an unreconstructed technophilia, and I will comment on that later. I should also indicate that some of what follows has been said elsewhere (King, 1992).

Perhaps I should begin, however, by declaring my position. I am an academic and administrator in distance education at the University of South Australia. Personally, as a long time member of the Labor Party, and with a perspective drawn from my own academic involvement in distance and open learning, I have some reservations about what the Government has embarked upon with the OLI, both in terms of its intentions and in the methods it has adopted to realise those intentions. However, as an administrator with some commitment to what my employer institution stands for as a 'new' university, I strongly believe that the OLI, having been established, should be supported and that the University of South Australia should strive to be a significant player within it, both through participation in its policy-making bodies and as a contracted supplier of course materials and student support services. From my perspective, any university which aspires to some more socially responsive role than that of the ivory tower must join with the OLI or watch from the sideline as it 
shapes and determines the tertiary education and training agenda for tens of thousands of Australians.

I am assuming that most here will have read something of the OLI in the press, but that not everyone will necessarily have a clear view of what the OLI proposal involves. Indeed, a number of the more excited press reports recently probably only add confusion to the issue.

This paper outlines the elements of the OLI, considers its historical roots, looks at some of the claims for and against the initiative, and attempts some cautionary generalisations.

\section{The Proposal}

Universities have been invited to bid for a national open learning initiative (OLI) in tertiary education. The stated purpose of the OLI is to widen and facilitate access to tertiary provision. This will involve off-campus courses for which there is evident high demand. Costs will be met by participants at a level equivalent to the Higher Education Contribution scheme.

The critical features of the proposal are:

1. an independent brokering agency to provide higher education

2. established by a university or group of universities

3. leading to degree courses

4. comprised of subjects from existing accredited programs

5. which would have parity with existing higher education degrees

6. to be available for people on payment of a fee and

7. to be set by DEET at about the level of HECs.

Senior DEET officials have indicated that the OLI is intended to become a third strand of tertiary education, to complement both TAFE and existing university programs. Minister Baldwin has represented it as a consolidation of existing distance education provision. It is intended as a low-cost option for which the Government will provide some start up costs from 1993 to 1995, after which the OLI is expected to be self-funding. Government support would not cover any capital component, nor would it be related to student numbers. Degree programs are to be delivered at a rate of $\$ 2400$ per year.

The Department expects a brochure to be ready by December 1992 and some 30 subjects available in 1993, many of which would be required by March. There would be an expectation of innovation and some television delivery, although this would not be the major component. Subjects taught through the OLI would be expected to have parity with subjects taught in conventional university programs. 
It should be understood that this initiative was proposed from within the University sector and has been shaped at all stages by the understanding that it has the support of at least two prominent institutions.

The OLI derives from a proposal initiated by the University of Queensland for a national distance provider, 'The University of Australia'. DEET responded to this by requiring the University of Queensland to negotiate a joint proposal with Monash University, which heads the successful TV Open Learning Project and to which my University is party. Monash and Queens-land made a joint bid to the Commonwealth, which Minister Baldwin announced to the press as a venture likely to be supported by the Government to about $\$ 39$ million. This aroused considerable response from the rest of the University sector, widely reported in the educational press, and the Government was obliged to call for general bids to provide the services proposed. A briefing was held for interested parties in Canberra. At that meeting, DEET representatives indicated that while the TAFE and private sector institutions had expressed considerable enthusiasm for the proposal, it was only open to universities to bid, although other institutions or systems could be partners to a university submission.

We now know that three bids were considered by the Evaluation Panel established by DEET to provide advice to the Minister: one from the University of Queensland, another from Monash University representing a consortium largely drawn from the earlier TV Open Learning Project partners, and a last minute bid from the combined Distance Education Centres (DECs) headed by University Partnerships, the entrepreneurial arm of the University of New England. The latter represented a counterMonash proposal, worthy in its own right, but directed to establishing a more participative management structure than the Monash bid intended. A number of universities had indicated they would cooperate with whichever party was successful.

I would like to cut through the weeks of plot and counter-plot, press leaks and institutional defections, backstabbing and misrepresentation which followed the initial bidding process. Ultimately, the University of Queensland withdrew, recognising it could not achieve support from the distance teaching universities. Monash and University partnerships were directed by the Evaluation Panel to achieve a compromise and this was not realised.

Minister Baldwin invited Monash University to form a company to develop and run the OU, subject to certain conditions. The most significant of these turns on the nature of the company board which would actually control the OLI. This was the matter during the competitive bidding process most at issue between Monash University and the other Distance Education Centres (DECs). Monash preferred a company which it 
controlled and for which it assumed all financial responsibility. Other DECs, through University Partnerships, sought equal representation of all participating universities on the company board. The Evaluation Panel did not accept that the University Partnerships proposal would provide efficient or sufficiently strong management although this was strongly contested by the DEC group.

The Minister has ruled that the OLI be managed by a company with a board of nine: four are to be Monash nominees, including the Chair; 3 are to be Vice Chancellors of other DECs; and the Minister will appoint two outside members. The method of selection of the three other DECs to the company board is not specified, although it would be reasonable to assume that DEET expects Monash to propose a mechanism to it for consideration.

Monash is also required to invite all other DECs and a number of other Universities and TAPE departments to contribute distance education subjects (in the form of course materials and tutoring services) to the OLI. All participating institutions will have equal representation on an Academic Board, which is to determine all academic issues. Monash University is now believed to have accepted these conditions.

It is my own opinion that the Monash company will take some time to be established and that interim arrangements agreed with DEET will need to be put in place alongside the longer term negotiations to ensure that the deadline of March 1993 for first offering of subjects can be met. Most probably, this will involve an extended role for the TV Open Learning group, as institutions already involved in the developmental work the OLI company will eventually subsume and expand upon.

Ultimately, the OLI may be known as the Open University of Australia.

\section{The origins of the OLI}

While it is undeniable that the initial proposal which led to the OLI came from the University of Queensland, the legitimation it received from Government, indeed the immediate and almost overwhelming enthusiasm it bestowed upon the project, provides some measure of how closely the Queensland proposal accorded with Commonwealth aspirations.

At base, the initiative has its roots in DEET and ministerial dissatisfactions with a creature of their own devising, the DEC system.

The rapid growth of distance education over the last decade $(56,928$ students in 1991; $10.6 \%$ of total enrolments) occurred largely in the former College Advanced Education sector, despite the longstanding involvement of a small number of universities in external studies programs. Typically, distance provision arose rapidly and unplanned from existing on-campus 
courses, often in business or teacher education, and usually in advance of the capacity or willingness of institutions to provide appropriate levels of infrastructure support. As a consequence, some of the courses available were of poor quality, duplicated provision elsewhere, and appeared to take little account of any sensible cost-benefit analysis. This brought distance education under the close scrutiny of the Commonwealth and led to a rationalisation which saw the establishment of eight national Distance Education Centres in 1989.

The Government sought to improve quality, reduce duplication, and address the question of costs by controlling the means of production of distance education courses. Institutions which wished to remain providers of external studies programs had to work through one of the eight Distance Education Centres (DECs) or lose funding. This produced a number of consequences, some of which we have only recently been able to see. My own impression is that the quality of course materials and services to students in distance programs has improved, a perception which is reinforced by my colleagues in other DECs but to the present has not been subjected to systematic investigation.

Second, there has been a significant increase in cooperation between major providers of distance courses, in exchanging course materials for similarly directed courses, in staff development, in research, and in serious attempts to act in concert on matters of advice to government and the advocacy of the field. I am involved in one example of collaboration which has demonstrated a number of the purposes sought by the Government in establishing the DECs. The University of South Australia and Deakin University offer a joint degree, the Masters in Distance Education, which was produced using the academic expertise of both institutions, has identical teaching materials, and cost each University half of what it would have to produce the whole program separately.

Third, distance education has been costed in an independent survey of the eight DECs conducted by a private consultant and funded by DEET. This produced strong evidence to show that distance education is not presently cheaper than on-campus provision to any significant degree, although the costs are differently constituted. Certainly, it provided no basis for reducing the funded load for distance students.

The DEC system, particularly the funding arrangements obliging other institutions to work through the Centres to develop distance teaching materials, was soon resented. First, it made all institutions face what teaching Students at a distance actually cost, where this had previously been largely hidden will incorporate overheads. Second, the actual funding arrangements involved non-DECs temporarily losing control of $15 \%$ of their external student generated funding, which involved real losses financially, in that short term investment to increase university 
revenue was not possible for the period involved. Third, some universities believed that the establishment of special infrastructure within the DECs, eg. video conferencing facilities, was denying them the opportunity of using teaching strategies which were generally desirable and could be employed outside the distance education field. Clearly, this is critical. The argument that concentration of technological infrastructure in the DECs prevents other institutions from engaging in technologically supported innovative teaching is serious and strikes against any policy desire to see more openness generally in higher education. Yet, there is some irony in the force this argument appears to have had. On the whole, apart from video-conferencing, there was little evidence that the DECs had received significantly larger resources for innovative delivery technologies than any other institutions. They had benefited from funds to trial technologies for the improvement of administrative infrastructure, eg voice-mail, but this support was not the target of the criticism. Further, as is taken up below, there appeared to be an apparently contradictory view in Canberra that the DECs were reluctant to move to technological innovations.

The Government appeared to lose its nerve about the large step it had taken by intervening in higher education to create the DEC system and there was talk of the need for reviews before the new arrangements had barely been implemented.

There seem to have been at least three other forces at work which also caused the Commonwealth to rethink its position on distance education. First, the educational debate was changing and 'open education' was seen as affording new opportunities for the various stakeholders in higher education, eg. employers, students, government and institutions. Second, there was increasing concern about the problem of unmet demand. Third, there was in increasing push within DEET and the Ministry to seek technological solutions to education problems. I should perhaps say something about each of these.

At the end of the 'eighties, there was considerable interest in the concept of open learning, both within individual institutions and amongst policy makers (See Johnson, 1990). There were two distinct common usages, which both confounded discussion and allowed some practitioners to assert values in their own programs which were not actually there. The first of these is summarised by Prof. Johnson (1990 p. v):

'Open learning' is a term increasingly used in educational writing. It embraces such practices as open admission or more flexible admission requirements to educational courses; student choice of topics and modes of study (face-to-face, distance/external, full-time, part-time); student choice of timing and manner of assessment; use of communication technology to facilitate choice and learning. It is more an approach to education than a particular technique. 
The critical element of this approach is the weight given to openness as flexibility, or the maximisation of student options. The second usage is more narrow, but borrows elements from the range of practices identified above, particularly the use of technology to deliver educational programs. This second sense is quite prevalent in TAFE circles and is often used to describe the delivery of negotiated courses to the workplace, often in consultation over content with employers and industry representatives. Commonly, developments in communications technology are used to enhance these arrangements. My concern about this latter use of 'open' is that whatever the other merits of these initiatives, sometimes the only flexibility involved is in the movement of courses off-campus and into the workplace. It is not unusual for all other aspects of the program to be quite constrained and to emphasise employer needs rather than student choice. In particular, I contend, the implied position sometimes taken by publicists of these ventures that use of non-print technologies for educational delivery of itself constitutes openness is an absurdity.

The importance of this for our purposes is that universities are seen as insufficiently 'open' in the first sense identified above by Commonwealth policy makers and, indeed, in many instances they are clearly not open in their practices and institutional arrangements. My own view is that the distance education component of higher education has been unfairly singled out as failing in this regard. This is in part because distance educators have been most inclined to pursue openness and are being judged as though their failure to escape entirely from the institutional constraints within which they operate was due to their lack of will rather than the force of convention operating within universities. Examples of individual instances of 'openness' in nondistance education settings were seen as evidence of the failure of distance educators and the DECs in particular. The mood at the present appears to be to denigrate distance education for failing to win on all fronts, rather than to support individual achievements of openness wherever they occur and support their wider application. DEET/ and Minister Baldwin, appear to have given up on distance education and be looking around for some other way of achieving open education.

The problem of unmet demand is a major concern of both Government and the universities. It is clearly related to the state of the national economy and the problem of widespread unemployment, particularly youth unemployment, but not only those things. It has direct links to calls for a 'clever country' and the capacities of individuals and groups to operate successfully within an increasingly technologically dominated international environment, rather than simply being controlled by it.

At present, unmet demand reflects the incapacity of Government to fund places for all those who qualify for university admission, including a significant number of mature age people. There is, for example, almost no 
projected growth in South Australian universities over the next three years, regardless of levels of demand. I have some sympathy for the Government in this. The Australian people are particularly reluctant to pay through taxation for services they generally desire. We wish as a nation to socialise our benefits and privatise our rights. Understandably, the Government is looking around for other means of achieving a solution to unmet demand and it appears to have seen at least a partial solution in a particular view of openness, to which I return in a moment.

Technological developments are transforming higher education, This view is widely shared. The Higher Education Council argued in December 1990:

The explosive development of the information technologies enables higher education to be, to a large extent, freed from location and time constraints. More mature participants as well as any disabled or disadvantaged by location or work conditions, will likely place the convenience of learning at a time and place of their choosing above the cost of acquiring the technology. It is certain that information technology will alter the delivery mechanisms of higher education and learning in the workplace will become more common as the methods develop.. Indeed, there is no reason why the techniques developed for distance or off-campus learning cannot be used for on-campus teaching. The [Higher Education] Council wishes to review the extent to which these techniques can be used as effective alternatives to the traditional lecture/tutorial methods. (Higher Education Council, 1990, p. 35)

It is worth noting the emphasis in this quotation on delivery and flexibility. Those involved in distance education would probably argue that technological developments have been much more significant in the last five years in relation to administrative support for students and in the production of teaching materials, but there is no doubt that some technologies will allow increased flexibility in the delivery of courses. Unfortunately, there does seem to be a largely undifferentiated view of technology in some policy-making circles, ie all technology is good, all technology increases flexibility, etc. Now this is simply not so. Videoconferencing and broadcast television, for example, impose constraints of time and place. Other technologies discriminate against those who do not have appropriate hardware to access them. This is not an argument against technology, or the imaginative use of specific technologies to meet particular learning needs, but one does need to recognise that some decisions in relation to the application of technologies to delivery of higher education taken recently by Government have more to do with political rather than educational ends.

The well-publicised success of the TV Open Learning Project, whereby the $A B C$ screens video material in support of a small number of first year level university units, is one such example. Nothing on offer in that project was not already available from various of the distance teaching universities in Australia. What was different was the level of publicity a Government 
initiative in education received through the medium of a highly visible broadcast television component. It is highly doubtful that the TV Open Learning Project is addressing unmet demand. Only $12 \%$ of respondents to a survey of TV Open Learning students indicated they had applied unsuccessfully for a university place (Keepes, 1992, p. 7). On the other hand, $83 \%$ of the respondents were over the age of 30, which is fairly typical of the student profile in distance education in Australia.

Nonetheless, the Government appears to see the TV Open Learning Project success as vindicating its criticisms of conventional on and off-campus university provision. It has drawn together its perceptions of that project as technologically innovative and therefore necessarily beneficial (albeit less than half- way through its initial trial) with its concerns for unmet demand and its criticisms of the inflexibility of present university provision in the formulation of its own requirements for the OLI.

\section{Costs and benefits of the OLI}

There have been reservations about the OLI expressed by representatives of the university sector, both to DEET and in the press. The most significant concern, in terms of institutional interests, is the level of fees allowed. It is clear that without significant economies of scale and some rationalisation of present distance education work practices, any university would have to reflect seriously on the cost-benefits of participation in the OLI.

Monash has undertaken a major analysis of the costs of the proposal and feels confident that it can assume financial responsibility for the brokering company over the ten year period required by the Commonwealth. In addition, the program areas on offer are in areas of very high demand, ie. Arts and Business, and most likely to allow economies of scale. Participating institutions will also discuss some rationalisation of work practices, most probably in the areas of:

1. sharing course development activity,

2. networking printing facilities,

13. inking warehousing and dispatch services to a central computer-based support system, and

4. simplifying credit transfer procedures.

There are other likely costs. One of some concern is whether student interests will be protected. In its original briefing document, DEET stressed the Importance of the OLI company providing adequate support for students and this was a matter pursued at length by the Evaluation Panel with the contending bidders. Monash has outlined its proposals in Volume 1 of its bid. 
Student representatives have also raised a range of issues about which they have concerns with the Minister.

There are two kinds of issue involved. First, there is the matter of whether the OLI as an initiative will alter the experience of study available to students in a way which disadvantages individuals or groups. This has been widely canvassed in the press and at student meetings. The second is whether the specific arrangements for management of the OLI take proper account of the needs of students and afford them adequate levels of support.

Given the establishment of the OLI, it appears the most productive course in relation to each of these is to seek participation in the decision-making of that body so that adequate measures are taken to maximise support for students and recognition of their concerns. Non-participation, or participation simply at the level of a contracted supplier of subject materials or other services, affords no opportunity to shape the way the OLI operates in its dealings with students generally.

The scale of the operation, with a reasonable projection of some 50,000 enrollees over the next three years, together with DEET's determination to create a third strand of tertiary education, strongly suggest that greater rather than less academic participation in its management affords the best opportunity for ensuring both responsible procedures and practices in dealing with students and protection of traditional university values and interests in the longer term.

The benefits the Commonwealth appears to see in the OLI are implicit in much of what has been said above. Some of these are quite political, which is hardly cause for comment. Others are more conventionally educational, or involve improvement to the delivery of higher education, and include:

1. provision of access to higher education which is open-ended, ie by avoiding conventional entry requirements, and broader, by drawing on a range of programs provided by different universities

2. creation of a variant of distance education which achieves economies of scale

3. development of an alternative to current provision which will be attractive to highly motivated mature age students.

Though reactions to the OLI proposal are mixed in higher education circles, the distance education community recognises that the initiative constitutes a major change to current patterns of course delivery and associated institutional practices. The choice for any university hosting a DEC is whether to be part of this change or to stand aside and observe its 
consequences. In considering the position of the University of South Australia, it became clear that the costs of non-participation were substantial, while involvement offered significant advantages.

Likely benefits to any distance teaching university from involvement in the OLI include:

1. the opportunity to influence decisions about the direction and methods of operation of the initiative

2. the provision of subject materials for OLI units for fee on a preferred basis

3. the provision of tutorial support for OLI units for which there staffing capacity within the University for fee

4. the likely extension of teaching materials in areas of academic strength through OLI subsidised television production

5. participation in innovations, eg. the use of voice mail for registration and enrolment, which have obvious potential application within the conventional operations of the University

6. association of the University with Government initiatives which may involve very large numbers of students and

7. recognition as an institution which has acknowledged and responded to the difficulties faced by those who cannot obtain conventional university places.

\section{Cautionary notes}

In closing, I would like to comment on a number of matters which concern me and to which attention must be paid. These are offset by observations on the positive aspects of the OLI.

\section{Costs}

Despite the work that Monash has done on this issue, I am still bothered that the OLI promises to deliver at a very small fee what is currently provided at full EFTSU (Effective full-time student units) funding. Despite the opportunities for economies of scale, it appears likely that some services will have to be cut and it would be most serious if these were the student support mechanisms which anecdotal evidence strongly suggests are linked to student retention in programs.

\section{Unrealistic expectations}

There has been far too much press comment of the "Sit in front of the TV and get a degree" kind. While it is important to attract attention to the initiative, there are dangers of quite unreal expectations being created by the way this new option for students is being presented. It is going to be 
quite difficult to pass sufficient subjects to qualify for a degree under the OLI, involving the exercise of considerable self-discipline and determination. In making education a marketable commodity, we should avoid the excesses of popular advertising.

\section{Attitudes to technology}

There appears to be a kind of cargo cult mentality growing around the OLI as though new applications of technology will afford magical solutions to longstanding problems of educational delivery to students at a distance. A recent press report suggested that by the end of the first year of the project some $70 \%$ of subjects would be delivered into students' homes using nonprint technologies. As anyone in this audience involved either in distance education or applications of communications technology to education understands, such statements are just nonsense.

\section{Quality of materials}

Some of the areas in highest demand for the OLI, eg. business subjects, are notoriously difficult to delivery through high quality materials at a distance. This is largely due to the lack of constancy of content and need for annual revision. This limits the capacity of the project to achieve economies through constant shelf-life of materials, although this may in part be overcome by developments in printing technology which allow much more flexibility in relation to revision. Nonetheless, the academic author's workload will still be present as an additional cost.

\section{Rate of implementation}

The obligation to delivery of OLI subjects by March next year does not take account of the time involved in establishing the necessary infrastructure. I have addressed this in part in suggesting that parallel developments in relation to materials production and establishing the OLI company may be necessary, but even so the time constraints seriously jeopardise quality in the first round of offer.

\section{Institutional cultures}

No attention has yet been paid to the changes in culture necessary at the supply end for the OLI to be implemented successfully. If academics, as they did with the TV Open Learning Project in my view, insist on treating OLI students with the same thoroughness as they do conventional distance education students, the costs of the project for individual institutions will increase substantially. The OLI demands that things be done differently, but the requisite change in academic and institutional culture has not been cultivated. 
However, these difficulties can be addressed and they need to be put into perspective. The OLI holds out a great deal to the Australian community. Academic institutions will also benefit if they choose to recognise the lessons the OLI affords.

\section{Increased places}

It seems likely that some 50,000 students may gain access to higher education subjects over the first three to five years of the initiative. This is as many as are currently served by the total university distance education community. Provided standards are maintained so the subjects on offer maintain parity of status with other university course components, this is an enormous benefit for the community at large.

\section{Rationalisation of effort}

The OLI will necessitate rationalisation of effort and the achievement of economies of scale which will allow deployment of energies into the production of learning materials with significantly enhanced quality.

\section{Credit transfer}

The problem of credit transfer which, to the present, universities appear almost incapable of resolving, should be substantially overcome by the agreement that Monash University, and perhaps other participating institutions, will recognise for degree granting purposes, all offerings of the OLI.

\section{Articulation between sectors}

The OLI will eventually offer both university and TAPE level studies. There is general agreement amongst parties that this must occur on the basis of planned articulation. Such ease of movement between awards at different levels is also in keeping with the general open nature of the initiative.

\section{Pioneering technological developments}

Because of its sheer size, the OLI will be able to employ technological developments more rapidly than can individual universities. For instance, it is likely to be the first Australian educational deliverer to have a substantial reliance on voice mail as a basis for all aspects of its student records generation.

\section{Powerful community presence}

The OLI, through both high visibility on TV and the establishment of local study centres, will increase the presence of higher education in the community at large. This is likely to be accompanied by increasing use of 
the resources of the OLI for informal education, e.g. by people simply watching educational television of high quality.

\section{Enhanced learning materials}

The system of subcontracting to competing institutions for the production of learning materials, the capacity to draw academic staff from different institutions to their authoring, and the resources available for quality presentation because of the scale of the undertaking should lead to a general improvement in the quality of educational resources generally available in the community, as occurred through the production of course materials at the Open University of the UK.

Benefits such as these allow me to return to the point made earlier. Regardless of one's reservations, the establishment of the OLI as fact enables us now to turn an educational problem to real advantage. It is my view that the effort to support the OLI as a worthy and defensible educational venture is both academically legitimate and in individual institutions' self-interest.

\section{References}

Higher Education Council, (1990). Higher Education: The Challenges Ahead, National Board of Employment, Education and Training. AGPS, Canberra, p.35.

Johnson, R. St. C. (1990). Open Learning: Policy and Practice. Commissioned Report No. 4, National Board of Employment, Education and Training. AGPS, Canberra, p.v.

Keepes, B., (1992). University Access via the Box, ACE News, 11(2), 7.

King, B. S. (1992). Right today but wrong tomorrow? The future for external, part-time and mature age students. A paper presented to the Conference Voices of Experience, External Student Union Inc., University of South Australia, at The Flinders University of South Australia, 20th July, 1992.

Author: Bruce King is an Associate Professor and Head of the Distance Education Centre at the University of South Australia, Holbrooks Road, Underdale, South Australia, 5032. This paper was his Keynote address to the Biennial ASET EdTech'92 Conference, 1-3 October 1992 in Adelaide. http:// www.ascilite.org.au/aset-archives/confs/edtech92/edtech92_contents.html

Please cite as: King, B. (1992). The open learning initiative: New directions for higher education. Australian Journal of Educational Technology, 8(2), 85-98. http:/ / www.ascilite.org.au/ajet/ajet8/ king.html 